\title{
Una biografía tecnológica de la industria
}

\section{Candolfi Arballo Norma ${ }^{a}$, Hualde Alfaro Alfredo ${ }^{b}$, Avitia Carlos Patricia ${ }^{c}$, Rodriguez Tapia Bernabéd}

${ }^{a}$ Universidad Autónoma de Baja California, BC, México, candolfi@uabc.edu.mx, ${ }^{\mathrm{b}}$ Colegio de la Frontera Norte, BC, México, ahualde@colef.mx, ' Universidad Autónoma de Baja California, BC, México, patricia_avitia@uabc.edu.mx, ${ }^{\mathrm{d}}$ Universidad Autónoma de Baja California, BC, México, rodriguez.bernabe@uabc.edu.mx.

\section{Resumen}

La presente investigación tiene por objetivo analizar el historial de aprendizaje de los líderes empresariales del sector productivo, denominada biografía tecnológica. La intención de la biografía tecnológica es el construir una ruta de aprendizaje sobre herramientas innovadoras de comunicación y colaboración de quienes están al frente de la industria.

La metodología de evaluación se define con un enfoque cuantitativo, orientado a la etnografía digital. Se realiza una intervención aplicando un instrumento de medición en base a la autopercepción de los líderes participantes. En el análisis se profundiza sobre las habilidades y la intensidad de uso de herramientas tecnológicas, mismas que se distinguen por brindar soluciones a las empresas, eficientando sus procesos internos y externos.

El caso de estudio se enfoca en la industria de las energías renovables, seleccionada debido al impacto del sector a nivel mundial y por considerarse uno de los principales gremios de desarrollo en México. Se estudian las pequeñas y medianas empresas en el estado de Baja California, ubicado en el límite territorial del norte de México, uno de los estados con mayor potencial para la generación de energía renovable en el país, debido a sus condiciones geográficas y naturales, con proyectos de energía solar, eólica, geotérmica, mareomotriz y de biomasa.

Los resultados de la biografía tecnológica dan evidencias positivas, debido a que exponen competencias tecnológicas a un nivel alto de los líderes respecto a la interacción con dispositivos y aplicaciones. Los líderes evaluados proyectan un grupo de empresarios con claridad en el autoaprendizaje constante. 
Palabras claves: Tecnologías en la industria, competencias tecnológicas, aprendizaje tecnológico, apropiación tecnológica.

\section{Introducción}

La industria en la actualidad se enfrenta al desarrollo tecnológico, a la innovación empresarial y a la revolución 4.0, dichas temáticas representan un desafío para establecer una visión de crecimiento en las organizaciones, sobre todo en países en vías de desarrollo (Carrillo \& Hualde, 2000; Carrillo \& Gomis, 2003; IMCO, 2006; AMITI, 2011; Ahumada, Zárate, Plascencia \& Perusquia, 2012; Buenrostro, 2013; Vázquez, Estrada, \& Reyes, 2014). Las empresas mexicanas invierten poco en tecnología (IMCO, 2006; IFT, 2018) en comparación con países desarrollados, lo que implica en primer lugar, ser un síntoma de que los empresarios mexicanos no tienen una clara percepción de las oportunidades de mejora que ofrecen las Tecnologías de la Información, Comunicación y Colaboración (TICC) en términos de productividad o eficiencia administrativa; en segundo lugar, apunta a un problema aún más complejo, en donde intervienen factores de índole económica, social, cultural y educativo, o bien, con capital humano que posee conocimientos de TICC a nivel bajo, así como un limitado acceso a infraestructura física y tecnológica, lo que justifica el incremento de la brecha digital en la industria mexicana.

La inequidad tecnológica en el país y el aumento de la brecha digital en la industria, dificulta el aprovechamiento de las TICC y los beneficios que ofrecen en las organizaciones, en ese sentido, resulta crucial avanzar en términos de cultura digital considerando el desarrollo de competencias tecnológicas en el sector productivo (Tyler, 2001; Nieto, 2004; Savory, 2006; Real, Leal, \& Roldán, 2006; Urraca, 2007). El desarrollo de competencias tecnológicas debiera basarse en el aprendizaje constante al interior de las organizaciones (Rojas, García \& García, 2011; Martín-Rojas, García-Morales \& MihiRamírez, 2011; Martín, García \& Bolívar, 2013), considerando las demandas internacionales sobre habilidades técnicas y tecnológicas (Youssef \& Dahmani, 2013; Lee, Park \& Lee, 2013; Motta, Zavaleta, Llinás \& Luque, 2013; European Commission, 2014) y entendiendo la relación de términos como competencia, capacidad y habilidad en un sentido tecnológico (Ordoñez, Gil-Gómez, Oltra \& González-Usach, 2015; Wilson, Leahy \& Dudley, 2015; Vallejo, Gallo \& Plazas, 2016; Candolfi, Chan \& Rodríguez, 2019).

Una vision tecnológica en las organizaciones, posibilita la adaptación a cambios desde la planeación organizacional, el aprovechado de las TICC e incursionar en el trabajo colaborativo (IFT, 2018). El crecimiento en la industria de las TICC considera tanto el desarrollo y producción de equipamiento e infraestructura, como la transformación de 
servicios para posibilitar la distribución global de productos (Hualde \& Díaz, 2010)(Carrillo \& Gomis, 2003). Sin embargo, las empresas mexicanas en su mayoría no cuentan con competencias tecnológicas que abonen ideologías innovadoras y se consoliden en corto plazo como un grupo de primer nivel, lo que limita su crecimiento a nivel nacional e internacional por tener desventajas competitivas (IFT, 2018), considerando que la manipulación de artefactos tecnológicos, así como la habilidad de comunicarse y colaborar con otros por medio de dispositivos electrónicos en acciones personales y laborales, es la base para el desarrollo de competencias tecnológicas de mayor profundidad.

\subsection{Competencias tecnológicas y su evaluación}

Las competencias tecnológicas en la industria se refieren a los conocimientos, habilidades y valores, como parte de un conjunto de disposiciones cognitivas relacionado al capital humano de una organización, con un dominio tecnológico que no se oriente únicamente a la incorporación y uso consciente de artefactos tecnológicos, si no, al desarrollo de una cultura innovadora, una apropiación inconsciente de las TICC (Candolfi, Chan \& Rodríguez, 2019). Una parte escencial en el desarrollo de competencias tecnológicas en el sector productivo, se refiere a la necesidad de identificar las técnicas de aprendizaje del capital humano y el conocimiento general con el que cuentan al relacionarse e interactuar con la tecnología de forma eficiente, en un inicio con equipo y herramientas de práctico uso, con la intención de elevar el dominio y la práctica de forma gradual.

La exploración de competencias tecnológicas se observa desde diversas áreas del conocimiento, como es la pedagogía, la economía, la administración, entre otras. Las metodologías aplicadas para su evaluación se orientan al análisis de docentes, estudiantes o personal de una organización. En (Candolfi \& Chan, 2017) se describen resultados sobre la evaluación de las competencias ya habilidades tecnológicas, mismas que se distribuyen en cinco categorías: desde una visión integradora, con un dominio artefactual-competitividad organizacional, con un dominio tecnológico-competitividad organizacional, con un modelado de pensamiento, y con una visión trascendente. En la presente investigación en particular, interesa la categoría sobre visión trascendente, en dónde González (1999) propone una metodología centrada en la historia de vida del sujeto, orientada a la noción de uso, y en dónde se documenta la autopercepción de las habilidades. La propuesta de González (1999) valora la génesis histórica, la habilidad de actuar con destreza en la con relación artefactos digitales prácticos y complejos, identifica las disposiciones de aprendizaje como durables en el tiempo, describe que las habilidades digitales son estructuras expandibles y tienen la posibilidad de transponerse.

Con el propósito de analizar a los líderes de la industria, el estudio se orienta a indagar sobre cuál es el conocimiento que tienen respecto al manejo de la tecnología, cómo han 
logrado aprenderlo históricamente y cuál es el nivel de habilidad que poseen, todo en un tono de autopercepción del participante. La intención es construir lo que se denomina una biografía tecnológica que representa una ruta de aprendizaje que siguen los líderes empresariales, al identificar los medios y las formas con que aprenden sobre herramientas tecnológicas, tanto de software como de hardware, así mismo, medir la habilidad y el tiempo de uso según aparatos electrónicos, de esta manera, se estudia el cómo aprenden tecnología, cuáles son las herramientas tecnológicas que más utilizan y cuál es el nivel de conocimiento de las herramientas tecnológicas.

Una biografía tecnológica posibilita la articulación de esfuerzos gubernamentales, educativos, empresariales y sociales para el mejor aprovechamiento de los recursos asignados a la promoción de las TICC en la industria, considerando la definición de políticas de desarrollo con mayor claridad, y así establecer propuestas de capacitación y actualización pertinentes.

\section{Enfoque de evaluación en el sector de energías renovables, la población objetivo}

El estudio se desarrolla en base a la metodología de la etnografía de la cultura digital y la innovación, bajo los enfoques de exploración de procesos de alfabetización y concentización digital, así como la participación social en procesos de innovación y tecnología. Se desarrolla una evaluación cuantitativa, integrada por un cuestionario de autopercepción que indaga sobre el histórico de aprendizaje del participante.

La evaluación es dirigida a los líderes del Sector de Energías Renovables (SER) del estado de Baja California, en el norte de México, en dónde el SER ha tenido gran proyección y se trabaja estratégicamente para su desarrollo y consolidación. Baja California es uno de los estados con mayor potencial para la generación de energía renovable en México, debido a sus condiciones geográficas y naturales, y actualmente se desarrollan proyectos relacionados con las energías solar, eólica, geotérmica, mareomotriz y de biomasa. El SER es un grupo de empresas relativamente nuevo en el estado, una novedosa y prometedora estrategia de inversión nacional, por ello, cuenta con gran impulso dentro de los planes de desarrollo y políticas empresariales. Las empresas del SER, en su mayoría se estructuran por un equipo de ingenieros expertos en las áreas de mecánica, eléctrica, electrónica y mecatrónica, orientadas principalmente a las actividades de capacitación técnica del personal, instalación de equipo eólico y solar, así como a la realización de proyectos a la medida de empresas ecológicamente responsables. 
Los participantes del estudio son 12 profesionistas que se desarrollan dentro del SER, denominados para el proyecto «líderes empresariales», los cuales se sitúan en un puesto con la responsabilidad de gerencia de una pequeña y mediana empresa. El grupo de empresas seleccionadas forman parte del registro de organizaciones de la Comisión Estatal de Energía en Baja California, definido como un sector emergente a nivel nacional.

\subsection{Descripción y validación del instrumento de medición}

Se integra un instrumento de evaluación con el objetivo de identificar el historial de aprendizaje de los líderes empresariales, el cuál se construye de seis dimensiones orientadas a medir el uso y el aprendizaje de diversos Dispositivos Tecnológico (DT), Aplicaciones Computacionales (AC) e Internet/Móviles (IM), en la Tabla 1 se describen las dimensiones e indicadores de evaluación. La evaluación se llevó a cabo por medio de la plataforma de Google forms de manera virtual.

Tabla 1 Descripción de las dimensiones e indicadores de evaluación

\begin{tabular}{|l|l|l|}
\hline Dimensión & Indicadores & Descripción \\
\hline Tecnologías & $\begin{array}{l}\text { 1. DT } \\
\text { 2. AC } \\
\text { 3. IM }\end{array}$ & $\begin{array}{l}\text { Equipo electrónico, lo relacionado con el software y } \\
\text { aplicaciones de Internet/móviles con aspectos novedosos } \\
\text { de redes sociales. }\end{array}$ \\
\hline Usabilidad & $\begin{array}{l}\text { 1. Utiliza el } \\
\text { dispositivo/software } \\
\text { 2. No utiliza el } \\
\text { dispositivo/software }\end{array}$ & $\begin{array}{l}\text { El líder identifica y responde al reactivo: ¿Sabe utilizar el } \\
\text { dispositivo/software? }\end{array}$ \\
\hline $\begin{array}{l}\text { Aprendizaje } \\
\text { 2. Capploración } \\
\text { 3. Formación profesional } \\
\text { 4. Indicar otra: }\end{array}$ & $\begin{array}{l}\text { El líder identifica y responde al reactivo: ¿Cómo aprendió } \\
\text { a utilizar el dispositivo/software? }\end{array}$ \\
\hline $\begin{array}{l}\text { Temporalidad } \\
\text { Ualor numérico }\end{array}$ & $\begin{array}{l}\text { El líder identifica y responde al reactivo: ¿Hace cuántos } \\
\text { años empezó a utilizar el dispositivo/software? }\end{array}$ \\
\hline $\begin{array}{l}\text { Habilidad de } \\
\text { uso }\end{array}$ & Valor nominal del 1-5 & $\begin{array}{l}\text { Frecuencia con la que se hace uso de los } \\
\text { dispositivos/aplicaciones. }\end{array}$ \\
\hline
\end{tabular}

Fuente: Elaboración propia

Para la validación del instrumento de medición se empleó la metodología de validéz de contenido por juicio de expertos (Lawshe,1975; Tristán, 2008; Díaz \& Baéz, 2015). Se definen seis jueces expertos en temáticas sobre estudios sociales en la industria, TecnoAntropología, liderazgo directivo, análisis e impulso de mercados, mercadotecnia digital, economía y gestión de la innovación y capacitación del capital humano. Se realiza la validación a través de una tabla de revisión, integrada por la dimensión de evaluación, el número de reactivo, el reactivo, la relevancia del reactivo - esencial, útil e inútil - , la 
claridad en la redacción del reactivo - legible o ilegible -, y las observaciones generales. Una vez realizada la validación, se calcula la Razón de Validéz de Contenido (CVR) y el Índice de Validéz de Contenido (CVI) utilizando el programa Microsoft Office Excel. Se obtiene el CVI de todo el instrumento validando para cada reactivo y dictaminando si es aceptable o inaceptable. En el resultados de la validación se esperan índices superiores a 0.5823, de lo contrario, el reactivo debe eliminarse del instrumento (Tristán, 2008). En la Ecuación 1 y Ecuación 2 se muestran las variables de la operación.

$$
\begin{aligned}
& C V R=\frac{n e}{N} \\
& \text { CVI }=\frac{\sum_{i-1}^{M} C V R i}{M}
\end{aligned}
$$

El resultado del CVI global del instrumento es de 0.83 , por lo tanto, se registra dentro de lo aceptable. Se obtuvieron observaciones sobre la redacción del reactivo, específicamente de la dimensión de dispositivos y aplicaciones, la dimensión de usabilidad y la dimensión de aprendizaje, los resultados se muestran en la Tabla 2.

\begin{tabular}{|c|c|c|c|c|c|}
\hline \multirow[t]{2}{*}{ Dimensión } & \multirow[t]{2}{*}{ Reactivo } & \multicolumn{3}{|c|}{$\begin{array}{c}\text { Relevancia } \\
\text { del reactivo }\end{array}$} & \multirow{2}{*}{$\begin{array}{c}\begin{array}{c}\text { Cálculo de } \\
\text { validación }\end{array} \\
\text { CVR } \\
\end{array}$} \\
\hline & & A & B & C & \\
\hline $\begin{array}{l}\text { Dispositivos y } \\
\text { aplicaciones }\end{array}$ & Lista de dispositivos y aplicaciones & 5 & 1 & 0 & 0.83 \\
\hline Usabilidad & ¿Sabe utilizar el dispositivo/software? & 5 & 1 & 0 & 0.83 \\
\hline Aprendizaje & ¿Cómo aprendió a utilizar el dispositivo/software? & 5 & 1 & 0 & 0.83 \\
\hline Temporalidad & $\begin{array}{l}\text { ¿Hace cuántos años empezó a utilizar el } \\
\text { dispositivo/software? }\end{array}$ & 5 & 1 & 0 & 0.83 \\
\hline Intensidad de uso & Evalúe su frecuencia de uso & 5 & 1 & 0 & 0.83 \\
\hline Habilidad de uso & Evalúe su habilidad de uso & 5 & 1 & 0 & 0.83 \\
\hline \multicolumn{2}{|r|}{ Suma } & 30 & 6 & $\mathbf{0}$ & 4.98 \\
\hline \multicolumn{5}{|c|}{$\begin{array}{lc}\mathrm{A}=\text { Esencial } & \text { CVI Global } \\
\mathrm{B}=\text { Útil } & \end{array}$} & 0.83 \\
\hline
\end{tabular}

Tabla 2 Descripción de reactivo

Fuente: Elaboración propia

\subsection{Fiabilidad del instrumento de evaluación}

Se considera el cálculo de Alfa de Cronbach para medir la fiabilidad del instrumento de evaluación, y se calcula la correlación de las dimensiones respecto a los dispositivos y aplicaciones (Prieto \& Delgado, 2010). Los resultados del cálculo de fiabilidad se obtienen utilizando el programa de estadística Statistical Package for the Social Sciences (SPSS) de IBM, los cuales se presentan en la Tabla 3 y la Tabla 4. 
Tabla 3 Calculo de Alfa de Cronbach

\begin{tabular}{|l|l|}
\hline Dispositivos y aplicaciones & $\begin{array}{l}\text { Alfa } \\
\text { Cronbach }\end{array}$ \\
\hline Equipo & 0.847 \\
\hline Software & 0.891 \\
\hline Web 2.0 & 0.865 \\
\hline \multicolumn{2}{|c|}{ Fuente: Elaboración propia }
\end{tabular}

Tabla 4 Análisis de correlación por reactivo

\begin{tabular}{|l|l|l|l|}
\hline Reactivo & Equipo & Sofware & Web 2.0 \\
\hline ¿Cómo aprendió a utilizar el dispositivo/software? & 0.644 & 0.758 & 0.48 \\
\hline ¿Hace cuántos años empezó a utilizar el dispositivo/software? & 0.848 & 0.657 & 0.673 \\
\hline Evalúe su frecuencia de uso & 0.625 & 0.858 & 0.936 \\
\hline Evalúe su habilidad de uso & 0.898 & 0.88 & 0.897 \\
\hline
\end{tabular}

Fuente: Elaboración propia

Los resultados del análisis de fiabilidad expuesto en la Tabla 3 demuestran una Alfa de Cronbach por encima del valor indicado como aceptable, por lo tanto, el instrumento de evaluación es confiable. En la Tabla 4 se observa que los reactivos con mayor puntaje tienen una contribución más directa al objetivo del estudio, lo que corresponde a la frecuencia y habilidad de uso de los equipos y aplicaciones, los reactivos con valores por debajo del 0.8 se consideran como datos que definen el contexto.

\section{Resultados}

En la dimensión de usabilidad, de los equipos como smartphone, computadora digital, tableta digitalizadora, así como aplicaciones de hojas de cálculo, correo electrónico y navegador de internet son las más utilizadas, el 100\% de los líderes mostraron conocimiento al respecto; el $91.6 \%$ de los líderes, respondieron que utilizan Smart TV, GPS, procesador de texto, programas de diseño y dibujo, así como bases de datos; en un nivel medio entre el $80 \%$ y $65 \%$ los líderes indican que utilizan i-POD/Mp3, i-POD/Mp4, software de edición de imagen, programas de facturación electrónica, programas de contabilidad de la empresa, programas de gestión y administración de proyectos, cuenta de LinkedIn y aplicaciones en la nube; y con valores porcentuales por debajo del $50 \%$, el uso de Kindle, Home feature, Smartwatch y Pen drive. En la Figura 1 se muestra los resultados en la dimensión de usabilidad.

En la dimensión de aprendizaje, los resultados exponen que la mayoría de los líderes en las diversas aplicaciones y equipo aprenden explorando por su propia cuenta, seguido de este valor que representa el $70 \%$, se encuentran en igual valor con un $15 \%$ la capacitación y 
actualización por medio de cursos y la formación profesional ya sea en licenciatura o posgrado. Los líderes empresariales proyectan un grupo con claridad en el autoaprendizaje constante;

\section{Figura 1 Resultados de usabilidad}

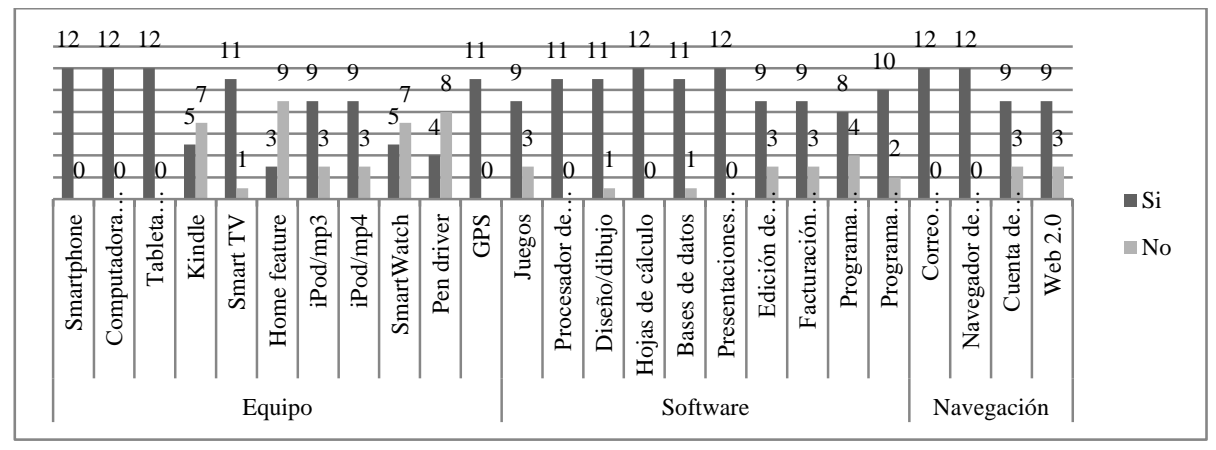

Fuente: Elaboración propia a partir de resultados

En la dimensión de temporalidad, se revisa los años que tienen utilizando cierto equipo o aplicación, que en su mayoría responde a sus incios en la formación universitaria. En la dimensión de habilidad de uso, no se identifican valores que fundamenten una relación directa con la apropiación tecnológica, es decir, a mayor dedicación en el uso, se logra un mayor dominio de las herramientas, sin importar el referente de iniciación del conocimiento.

\section{Conclusiones y discusión}

Los resultados de la evaluación son positivos, debido a que los líderes muestran un alto conocimiento de las aplicaciones y el equipo que se utiliza tanto en la industria como en actividades de la vida diaria. En general, los líderes demuestran una biografía tecnológica con conocimiento medio, dispuestos a la actualización, con habilidades en herramientas digitales comunes. Sin embargo, se detectan oportunidades de mejora en la visión digital dirigida a lo administrativo, en donde se potencializan los procesos de desarrollo de proyectos, comunicación interna y externa, relación con clientes y proveedores por medio de la implementación de TICC con una frecuencia y habilidad de uso con mayor impacto. Se requiere que la apreciación tecnológica se convierta en un proceso transparente en las actividades laborales y personales del día a día. El presente estudio se desarrolla como un acercamiento al conocimiento y a las habilidades tecnológicas que los líderes de la industria poseen, analizando la manipulación de artefactos básicos y las potencialidades del sector a partir de una visión tecnológica de sus representantes. 


\section{Referencias}

Adamson, K. A., \& Prion, S. (2013). Reliability: measuring internal consistency using Cronbach's a. Clinical Simulation in Nursing, 9(5), e179-e180.

AMITI.(2011). Alianza Mundial de Servicios de Tecnologías de la Información. Recuperado: 2 de mayo del 2016 en: http://amiti.org.mx/

Ahumada, E., Zárate, R., Plascencia, I., \& Perusquia, J. (2012). Modelo de competitividad basado en el conocimieto: El caso de las PyMEs del Sector de Tecnologías de la Información en Baja California. Revista Internacional Administración \& Finanzas , 13-27.

Buenrostro, M. E. (2013). Experiencias y desafíos en la apropiación de las TICs por las PyMEs Mexicanas - Colección de Memorias de Seminarios. (D. ㅇ. Industria, Ed.) México: INFOTEC.

Candolfi, N \& Chan, M.E.(2017). Construcción conceptual de las competencias tecnológicas a partir de un análisis cronológico de su significado y su aplicación. De lo educativo a lo empresarial. En Cruz Flores, R.G. \& López Morteo, G.A. (Pearson Ed.), Tecnologías emergentes en la educación: El ámbito educativo en la era digital. México.

Candolfi Arballo, N., Chan Núñez, M., \& Rodríguez Tapia, B.(2019). Technological Competences: A Systematic Review of the Literature in 22 Years of Study. International Journal Of Emerging Technologies In Learning (IJET), 14(04), pp. 4-30. http://dx.doi.org/10.3991/ijet.v14i04.9118

Carrillo, J., \& Gomis, R. (2003). Los retos de las maquilladoras ante la pérdida de competitividad. Comercio Exterior, 53, 318-327.

Carrillo, J., \& Hualde, A. (2000). El desarrollo regional y la maquiladora fronteriza: Las peculiaridades de un cluster electrónico en Tijuana. Mecado de valores , 10, 45-56.

Díaz, Y. E., \& Báez, L. L. (2015). Exploración de la capacidad de liderazgo para la incorporación de TICC en educación: validación de un instrumento. RELATEC: Revista Latinoamericana de Tecnología Educativa, 14(3), 35-47.

European Commission. (2014). e-Skills: The international dimension and the impact of globalisation. European Commission DG Enterprise and Industry.

González, J. A. (1999). Tecnología y percepción social evaluar la competencia tecnológica. Estudios sobre las culturas contemporáneas, V (9), 155-165.

IFT. (2018). Adopción de las TIC y uso de internet en México. México: Instituto Federal de Telecomunicaciones.

IMCO. (2006). Visión México 2020. Políticas Públicas en materia de Tecnologías de la Información y Comunicaciones para impulsar la Competitividad de México. (I. M. Competitividad, Ed.) México: Concepto Total S.A de C.V.

Lawshe, C. H. (1975). A quantitative approach to content validity. (D. 10.1111/j.17446570.1975.tb01393.x, Ed.) Personnel Psychology, 563-575. 
Lee, H., Park, J., \& Lee, J. (2013). Role of leadership competencies and team social capital in IT services. Journal of Computer Information Systems, 53(4), 1-11.

Martín-Rojas, R., García-Morales, V. J., \& Mihi-Ramírez, A. (2011). How can we increase Spanish technology firms' performance?. Journal of Knowledge Management, 15(5), 759-778.

Martín, R. R., García, M. V., \& Bolívar, R. M. (2013). Influence of technological support, skills and competencies, and learning on corporate entrepreneurship in Europa technology firms. Technovation , 417-430.

Motta, J. J., Zavaleta, L., Llinás, I., \& Luque, L. (2013). Innovation processes and competences of human resources in the software industry of Argentina. Revista CTS, 24, 147-175.

Nieto, M. (2004). Basic propositions for the study of the technological innovation process in the firm (Vol. 7). European Journal of Innovation Management.

Ordoñez, J. E., Gil-Gómez, H., Oltra, B. R., \& González-Usach, R. (2015). Importancia de las competencias en tecnologías de la información(e-skills) en sectores productivos. Propuesta de investigación en el sector transporte de la comunidad Valenciana. 3Ciencias TIC , 4 (12), 87-99.

Real, J. C., Leal, A., \& Roldán, J. L. (2006). Information technology as a determinant of organizational learning and technological distinctive competencies. Industrial Marketing Management, 505-521.

Rojas, M., García, M. V., \& García, S. E. (2011). The influence on corporate entrepreneurship of technological variables. Industrial Management \& Data System, 111 (7), 984-1005.

Savory, C. (2006). Translating knowledge to build technological competence. Management Decision, 44(8), 1052-1075.

Tristán, A. (2008). Modificación al modelo de Lawshe para el dictamen cuantitativo de la validez de contenido de un instrumento objetivo. Avances en medición, 37-48.

Tyler, B. B. (2001). The complementarity of cooperative and technological competencies: a resourcebased perspective. Journal Engineering and Technology Manegement Jet-M , 18, 1-27.

Urraca, R. A. (2007). Patrones de inserción de las empresas multinacionales en la formación de competencias tecnológicas de países seguidores. Revista Brasileira de Innovación , 405432.

Vallejo, B. M., Gallo, J. J., \& Plazas, C. E. (2016). Propuesta de una metodología para el estudio de competencia tecnológica en empresas fabricantes de medicamentos. Universidad \& Empresa , 18 (31), 11-27.

Wilson, D., Leahy, D., \& Dudley, D. (2015). The European e-competence framework: Past, Present and Future. IADIS International Journal on Computer Science and Information Systems , 10 (1), $1-13$. 